\title{
Traditional Ecological Knowledge in Connection with Non-Domesticated Animals in the Slovenian and Hungarian Borderland
}

Received: May 29, 2020 - Accepted: September 2, 2020

\author{
Viktor Ulicsni - Dániel Babai \\ Institute of Ecology and Botany, Centre for Ecological Research, Vácrátót (corresponding author) - \\ Institute of Ethnology, Research Centre for the Humanities, Budapest
}

\begin{abstract}
Although a significant proportion of folk knowledge of nature concerns knowledge of invertebrates and vertebrates living in the wild, very little ethnozoological research has been carried out in Central Europe focusing on the whole fauna. In writing the present paper, our aim was to contribute to filling this gap by interviewing 40 local farmers who are particularly knowledgeable on this topic, half of them from the Örség region of Hungary, and half from the neighboring villages in Slovenia, and by recording their knowledge with respect to nondomesticated animals.

Our research identified the second highest number of taxa (242 species-level folk taxa) in terms of investigations carried out in the Hungarian language area in relation to the entire fauna. These included 129 invertebrate folk taxa, $73 \%$ of which were called by a species-specific name. They also included 109 vertebrate folk taxa, 103 of which had a separate species-level local name. In the case of two groups (butterflies and mammals), we also investigated attributes that were important and salient from the point of view of species knowledge: morphology and size were of particular relevance in relation to mammal species; while salient habitat features and frequency were relevant in relation to butterfly species. In the case of both groups, usefulness was the least important factor.

Despite the general erosion of traditional ecological knowledge in Europe, these recently collected data indicate that a rich, vibrant knowledge is still to be found among the Hungarians whom we interviewed in the Örség region and the neighboring villages in Slovenia. The especially large number of recorded folk taxa, and the accurate knowledge required to differentiate between them confirm, that even today it is worth carrying out investigations on this topic in East Central Europe in the interests of obtaining knowledge of, and conserving cultural and natural values.
\end{abstract}

Keywords: ethnozoology, folk taxonomy, Örség, species knowledge, traditional ecological knowledge (TEK), borderland 


\section{INTRODUCTION}

A knowledge of biodiversity - the plants, animals, and fungi living in the wild — has always been indispensable for the efficient use of resources in farming communities and for sustainable lifestyles throughout generations (BERKES 2012). In most instances, individual species are differentiated and classified according to three main principles: the animals' morphological appearance (e.g. size, shape, color); their ecological salience (e.g. behavior); and their cultural significance (in particular, their supposed or actual usefulness or harmfulness) (HUNN 1982). Likewise, according to HunN (1999), the morphological point of view, including physical size, along with the potential harm an animal might do, are the most important factors in relation to the extent of species knowledge.

However, as a result of rapid urbanization throughout the world and the disappearance of daily contact with the natural environment, this knowledge is becoming less and less important and will eventually be lost. Despite this, folk knowledge of wild animals is generally a less popular topic that is investigated by only relatively few people, mainly with a focus on the tropics and on vertebrate species, along with their uses (e.g. CosTANeto 1998; KutaleK - Kassa 2005; Walker 2000; EstabrooK 2008). Works that describe large numbers of folk animal species also chiefly concern vertebrates (REA 1998, 2007; DiAMOND - BISHOP 1999), and only a very few works have been published on invertebrate fauna in its entirety, for example in Honduras (BENTLEY - RODRÍGUEZ 2001), Tanzania (Hemp 2001), and Kenya (WePuKhUlu 1992). Investigations in Europe also tend to concentrate on smaller taxonomic groups, or a few prominent species (e.g. Benítez 2011; Svanberg 2006; Lescureux 2010; Ceríaco et al. 2011). Another area of investigation is the semiotic characterization of invertebrates (especially in terms of predictions related to the weather) (e.g. COSTA-NETO 2006).

In Eurasia, remarkably few works have been written dealing with many animal species simultaneously. The most comprehensive study is a book by Fridell and Svanberg, published in 2007, on Swedish ethnozoological knowledge. Ceríaco likewise examines a larger number of taxa in his work on amphibians and reptiles (CERÍACO 2012).

In the Hungarian language area, also, there are only a few examples of research on folk knowledge in relation to the whole fauna (Kovács 1987 - Felső-Szigetköz, Hungary; GuB 1996 - Sóvidék, Romania). Invertebrates are particularly underrepresented in ethnozoological studies, since research in this field demands a species knowledge that often goes beyond the erudition of ecologists not specializing in the given taxon group (e.g. in the case of the order Diptera, the number of species known to the local population even exceeds the number required in university ecological training [ULICSNI et al. 2016]).

The goal of the present work was to contribute to filling this gap by interviewing 40 local farmers who are particularly knowledgeable on this topic, half of them from the Örség region of Hungary and half from the neighboring villages in Slovenia, and recording the part of their knowledge related to non-domesticated animals. Although our work can in no way be regarded as exhaustive, it enumerates the second largest number of taxa (242 species-level folk taxa) compared to studies that have been carried out in connection with knowledge of the whole fauna in the Hungarian language area. The only work that describes larger numbers of species is the volume by GuB, published in 1996 in relation to Sóvidék, Transylvania, where 335 folk taxa were recorded from interviews with 307 inhabitants in 14 settlements. 
The data collection took place among people of Hungarian nationality and Hungarianspeaking interviewees involved in extensive agriculture in villages in Slovenia and Hungary (in the territory covered by the Örség National Park and the Goričko Natural Park). Since the interviewees spent (spend) a lot of their time in agricultural areas and forests in connection with their daily activities, their relationship with the natural environment was (is) both regular and immediate (Ispán et al. 2018). This has been the case for many generations. As a result, besides personal experience, a significant proportion of their knowledge has also been passed down (Hoppál 1982). Alongside personal experience and handed-down knowledge, only a very little of their knowledge comes from academic teaching or the reading of specialist literature (BABAI et al. 2019).

In relation to the non-domesticated animal species in the vicinity of the settlements and in the villages themselves, we investigated which species were known to our interviewees, which folk taxa they identified, and which names they used. Our preliminary hypothesis was that, despite the significantly different forms of land use that followed the defining of the boundaries in the 1920s, the basic knowledge of the population, who had been engaged in relatively homogeneous farming activities, would remain relatively similar, and that, as a result, we would record a largely similar knowledge of species. Among other things, we based our hypothesis - that two-thirds of the knowledge of species would be predictable and common - on the fact that the area's natural geographical and ecological relations did not change along with its borders, and that earlier investigations in the case of distant regions of the Carpathian Basin characterized by significantly different land use also indicated a significant proportion of common species knowledge (ULICSNI et al. 2016, 2019). In some cases, traditional ecological knowledge is rather conservative: there are many mechanisms in its operation that slow down the pace of change (HewletT - CAVALLI-Sforza 1986), and it typically survives and is handed down over centuries (ZeNT 2013). Besides, even if the frequency of an individual species has changed to a significant extent on the two sides of the border in the past one hundred years, the species composition, by contrast, has been less influenced by changes in land use. Our further aim was to explore the potential impacts that nature conservation activities, carried out with significantly different degrees of intensity, and the changes that have taken place in land use in the two countries, have had on species knowledge. For this purpose, besides the documentation of folk taxa, in the case of two taxon groups (butterflies and mammals) we also explored the reasons behind the knowledge of them.

\section{MATERIAL AND METHODOLOGY}

\section{The examined areas}

The examined areas, which are essentially hilly landscapes on both the Slovenian and Hungarian sides of the border (between 190 and $380 \mathrm{~m}$ above sea level), are characterized by a moderately continental, mildly subalpine climate. The average temperature varies between 9.1 and $9.8^{\circ} \mathrm{C}$, and the annual amount of rainfall is between 760 and $800 \mathrm{~mm}$ (DöVÉNYI 2010), approximately $600 \mathrm{~mm}$ of which falls in the vegetation periods (HAHN et al. 2012). 
In terms of the predominant, primary vegetation cover, deciduous oak and beech forests are typical of the enclosed mountains (hornbeam and oak forests: Quercus petraea - Carpinetum; beech forests: Fagion illirycum), where, alongside the dominant sessile oak (Quercus petraea), the pedunculate oak (Quercus robur), beech (Fagus sylvatica), and hornbeam (Carpinus betulus) are also to be found in large numbers. However, anthropological land transformation activities have had an enormous impact on this primary vegetation cover, resulting initially in a significant increase in the proportion of open, grassland areas, and subsequently in substantial areas of the landscape being given over to arable farming (BARTHA 2016).

Alongside the majority of the fauna that is typical of a moderately continental climate, subalpine animal species are also present in the area. These include many protected species that are valuable from a nature conservation point of view, which are connected with agricultural cultivation on small plots, a form of farming that has been abandoned to a significant extent throughout Europe. As one of the best-preserved cultural landscapes in Central Europe (KALIGARIČ et al. 2008), the Örség region and Goričko play an important role in the protection of these species. The approximately 1,500 species of Lepidoptera found in the region (VÍG 1998) and the number of dragonfly species at regional level in Hungary, are the highest here, thus nature conservation attracts particular attention (AmBrus et al. 1995; Víg 1998). Among the vertebrates, the highly protected Corncrake (Crex crex), the Eurasian otter (Lutra lutra), and the Italian crested newt (Triturus carnifex), a subalpine animal species that reaches the easternmost limit of its spread in this area, are of particular significance (VÍG 2003).

\section{Material and methodology}

Data collection took place in Slovenia in April ( 3 fieldwork days) and July ( 8 fieldwork days) 2018, and in Hungary in June 2019 (10 fieldwork days). The number of interviewees in each individual settlement in Slovenia was as follows: Prosenjakovci (Pártosfalva) - 9; Središče (Szerdahely) - 6; Motvarjevci (Szentlászló) - 3; Hodoš (Örihodos) - 1; Ivanjševci (Alsójánosfa) - 1. In Hungary, the distribution was as follows: Öriszentpéter - 7; Nagyrákos - 5; Szalafö - 3; Kerkáskápolna - 2; Kisrákos - 1; Viszák -1 ; Magyarszombatfa -1 . The average age of the interviewees was 75 in Slovenia (the youngest being 53 and the oldest 92); and 79 in Hungary (where the youngest was 67 and oldest 91).

Our objective was not to record average knowledge, but to identify those people with the greatest knowledge on this topic in the two regions. The knowledge held by these particularly knowledgeable interviewees encompasses the vast majority of the knowledge held by local people with average or little knowledge of the subject (ULICSNI et al. 2020), thus, if we are primarily studying overall knowledge, as in the present paper, it is more efficient to interview these people. Among the interviewees, only one from the Örség region had completed secondary school and one interviewee from Slovenia had completed college: the proportion of animal knowledge obtained by means of education was insignificant among the interviewees.

The data collection was carried out in Hungary using a snowball sampling method, starting from the best data providers identified on the basis of earlier investigations into 
traditional knowledge, performed by a local nature conservation engineer and the head of the Nature Conservation Department at the Örség National Park. In Slovenia, data collection was also carried out using snowball sampling, beginning with data providers identified by the director of a documentary film on the knowledge and customs of the local population, and a Reformed Church pastor. We informed the interviewees in advance about the research and about the goal of the interviews, and we made audio recordings only if prior permission was given.

The principal means of data collection were indoor interviews (30 hours in Slovenia, 32 hours in Hungary), which were recorded by dictaphone. These interviews comprised semi-structured elements and free-flowing conversation, by means of which, in the first round, it was possible to perform identifications of species-level folk taxa. A collection of pictures of the species occurring in the examined region contributed to accurate identification (10 to 15 photographs of various different species were printed on one A4 sheet). In cases where conflicting descriptions/profiles were provided by the interviewees, the classification was confirmed by means of cross-questions aimed at specific characteristics that would accurately identify the given taxa.

In the case of the two groups that were studied in detail (butterflies and mammals), we investigated those characteristics that were found to be most important from the perspective of salience in the course of studies of the whole fauna (e.g. HUNN 1999; ELLEN 2006; ULICSNI et al. 2019). The investigation of these aspects is particularly important, because, among other things, the (at least partial) investigation of the reasons behind species knowledge contributes to an understanding of the manner in which knowledge is constructed; recognizing the most important perspectives from the point of view of the local population contributes to a knowledge of their world view; and, especially in the case of key species, it can help us in the management of potential (nature conservation) conflicts. The 10 most important aspects in terms of salience were the following: physical size, morphological salience, ethological characteristics, frequency, habitat, danger to human beings, harmfulness, usefulness, richness of folklore, and significance in terms of nature conservation. In the case of each of these aspects, we established six categories to express the relationship between the given species and the local human population ( 0 : no relationship; 1 : species with little significance -5 : species with great significance).

In determining the size of the species, we took relative size into account separately within the butterflies and mammals. In the event of uncertainty, we took as the basis the ontogenetic phase with which members of the local population were most likely to be familiar. In the case of morphological salience, we divided the species into five categories on the basis of conspicuous color, the special nature of the surface/body (typical, striking, unusual, strange), and difference from the average (rounded) morphology. Ethological characteristics were assessed on the basis of sound, smell, agility, and conspicuousness. In determining the prevalence of individual taxa, we took into account their local prevalence, based on our own experience and the scientific literature (BÁLINT 1994; AmBrus et al. 1995; VíG 1999, 2003). In defining habitat, we took into consideration the possibilities for, and frequency of, encounters with human beings. The degree of danger to human beings ranged from being a minor nuisance to creating intolerable disturbance, and even causing death. Harmfulness and usefulness were exclusively related to damage or benefits in relation to domestic animals, cultivated plants, or other human property. By means of the folklore/attitude category, we studied subjective relationships with the 
examined animal species, as well as the diversity of their appearance in the folklore genres of the rural and urban environment. When classified according to their significance for nature conservation, the species were evaluated as follows: 0: alien or indigenous species needing to be culled; 1: indigenous species that occasionally cause damage, that are neither protected nor endangered; 2: species that require protection, that are not endangered or that are only slightly endangered, and that cannot be legally hunted; 3: species accorded protection as hunting assets or for other reasons; 4: vulnerable species that are officially protected in Hungary; and 5: highly endangered species that enjoy a high level of protection.

Uncertainties in classification were eliminated by means of iteration. In the case of each individual species, we surveyed the related literature. The first classification was performed by Dániel Babai and Zsolt Molnár, who did not participate in the ethnozoological collection; and the two authors (with the help of Zsolt Molnár) worked on the finalization of the scores, which we accepted only in the case of unanimity. We added together the given scores in the different categories (0-6) by aspect (e.g. size, usefulness, etc.), and then compared these scores.

\section{RESULTS}

In the examined communities, we successfully classified a total of 242 scientific taxa that could be identified with species-level folk taxa. Fifty-five percent of the taxa - that is, 133 taxa, were invertebrates, while 109 (45\%) were vertebrates (Table 1). The number of folk species known in Hungary and Slovenia was largely the same (210 and 202 respectively), and the proportions of species likewise differed only slightly: In Slovenia, vertebrates amounted to 49\% (98 taxa), while in Hungary they amounted to 45\% (94 taxa), compared to 104 and 116 invertebrate taxa respectively. With respect to mammals, the same 28 species were known in the two regions, while in the case of invertebrates, we discovered a relatively large number of taxa that were known in only one of the regions (35\%, 46 taxa). Out of the 242 taxa, $162(67 \%)$ could be associated with biological

Table 1. The proportion of scientific taxa belonging to different taxonomic groups that can be identified with species-level folk taxa in the examined groups

\begin{tabular}{|lcccc|}
\hline taxonomic group & $\begin{array}{c}\text { mentioned } \\
\text { exclusively in } \\
\text { Slovenia }\end{array}$ & $\begin{array}{c}\text { mentioned } \\
\text { exclusively in } \\
\text { Hungary }\end{array}$ & $\begin{array}{c}\text { mentioned in } \\
\text { both countries }\end{array}$ & total \\
\hline mammals & 0 & 0 & 28 & 28 \\
\hline birds & 10 & 9 & 38 & 57 \\
\hline reptiles & 0 & 0 & 7 & 7 \\
\hline amphibians & 1 & 2 & 6 & 9 \\
\hline fish & 4 & 0 & 4 & 8 \\
\hline invertebrates & 17 & 29 & 87 & 133 \\
\hline total & 32 & 40 & 170 & 242 \\
\hline
\end{tabular}


species, and 76 were folk species that embraced several biological species, while in a few instances (4 folk species, $2 \%$ ), sub-scientific, over-differentiated species were also found. A folk classification that did not fit precisely into this scientific system, or that went beyond the scientific taxonomy, was identified in the case of the Northern whitebreasted hedgehog (Erinaceus roumanicus). In this case, a so-called dog-like or pig-like taxon was distinguished within the species in both regions.

In the case of mammals, the most important salient features in terms of species knowledge were morphological salience and size (highest total salience values, see Table 2), although frequency, ethological salience, and habitat salience came closely behind this value. By contrast, in the case of butterflies, habitat salience and frequency counted as the key aspects, although alongside these, morphology, ethological salience, and size were likewise important in relation to knowledge of species (Table 3). Harmfulness played a medium role in the case of both taxa in relation to species knowledge, while, by contrast, usefulness counted as the least important distinguishing feature in the case of both groups. Importance in nature conservation and folklore/attitude were of small to medium significance in both groups in terms of distinguishing among the taxa.

From the list of folk invertebrate taxa known to the interviews, most of the species were referred to by name (129 taxa, 97\%), and of these, 90 species (68\%) were called by a special, species-specific name (Table 4). The list contains quite a few species, which, even though they have a simple name that is occasionally also used as a folk taxonomic (folk generic) category, nevertheless also bear the same name within a smaller taxonomic category, as a type species or as a species name in its own right. This situation is often justified by the addition of the epithet "common/true/real" (e.g. the house fly: Musca domestica; the turf or pavement ant: Tetramorium caespitum and similar species; the clothes moth: Tineola biselliella; etc.).

The arrangement used in the table, according to scientific taxonomic classification, largely corresponds with the folk classification, as clearly illustrated by the folk names (e.g. spiders among the arachnids). At the same time, there are several significant differences between the two systems, such as, for example, in the case of pond skaters (Gerris spp.), which belong among the so-called shield bugs, being classified under the folk spider taxon (this is evidenced by one of its folk names: water spider).

We also recorded a folk taxon known by the name lobodár, which belongs to the arthropod family and which we were unable to associate with a scientific taxon despite repeated and detailed questioning. The so-called házi kigyó ("house snake") is another of the taxa that we were unable to identify accurately with a scientific taxon (Table 5). With the exception of the lobodár, no description is given of obscure taxa that we were unable to identify accurately. The majority of taxa omitted from the classification for this reason were birds (Table 6). 


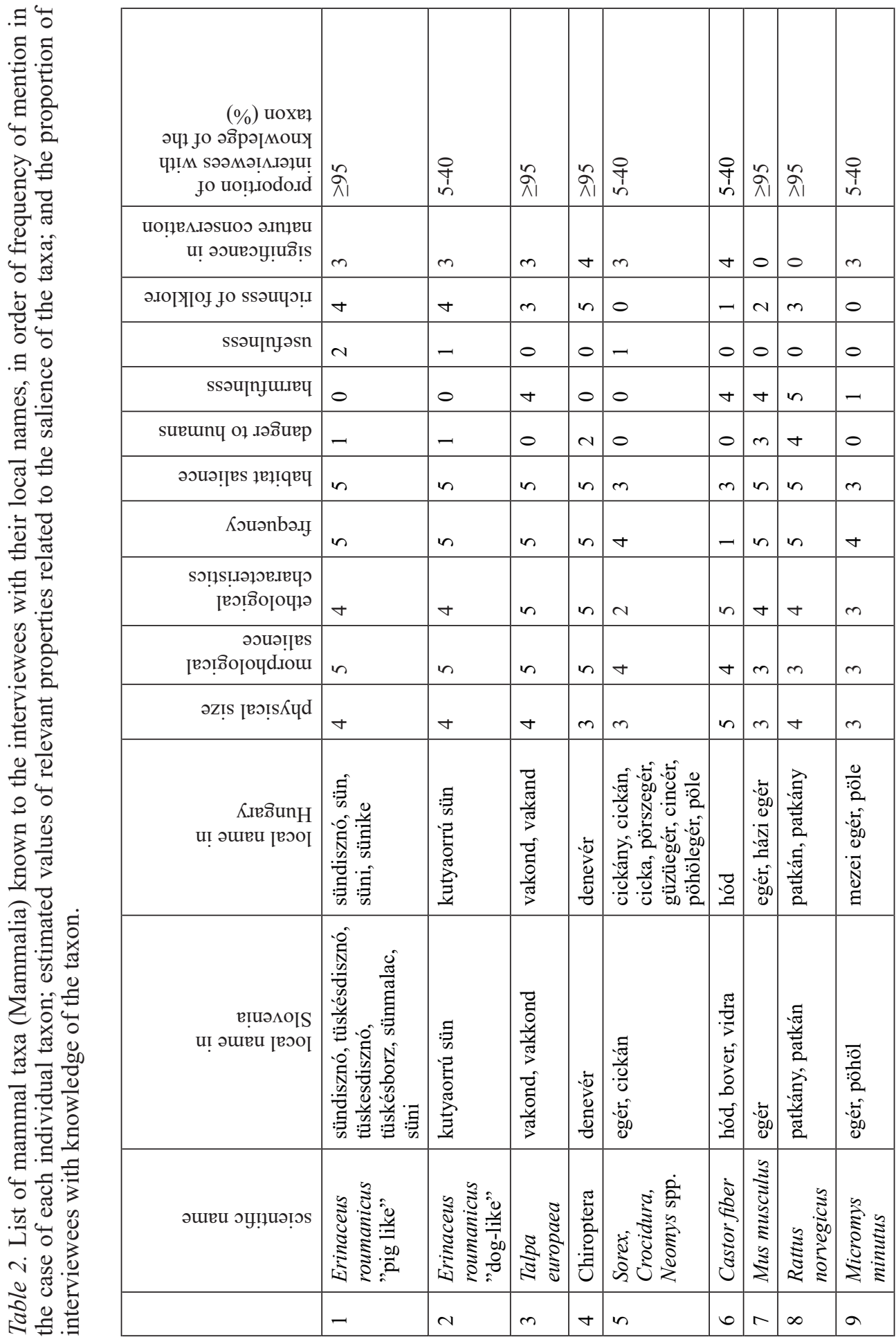




\begin{tabular}{|c|c|c|c|c|c|c|c|c|c|}
\hline 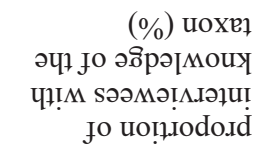 & 吕 & $\mathscr{v}$ & $\begin{array}{l}a \\
\hat{d} \\
\dot{b}\end{array}$ & $\mathfrak{v}_{1}$ & $\mathscr{V}$ & $\begin{array}{l}\text { O } \\
\stackrel{1}{+} \\
\text { Pे }\end{array}$ & 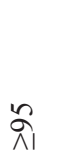 & $\stackrel{\varkappa}{\hat{\Lambda}}$ & ầ \\
\hline 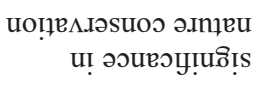 & - & - & $m$ & 0 & $\nabla$ & $\nabla$ & $m$ & $m$ & - \\
\hline 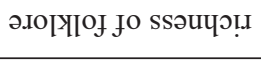 & 0 & 0 & 0 & 0 & 0 & 0 & $m$ & N & 0 \\
\hline ssəu|nృəsn & 0 & 0 & 0 & - & 0 & 0 & 0 & $m$ & - \\
\hline ssәu[пушиеч & $\nabla$ & 0 & $m$ & 0 & $\sim$ & - & - & $m$ & $\nabla$ \\
\hline suruny ol .əəoินep & 0 & 0 & $m$ & 0 & 0 & 0 & 0 & 0 & 0 \\
\hline 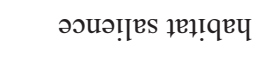 & $\theta$ & $N$ & $\forall$ & $\sim$ & $\sim$ & - & $m$ & $\nabla$ & in \\
\hline Кouənbə.I & in & $\nabla$ & $n$ & - & $m$ & $m$ & $\nabla$ & $\nabla$ & $\nabla$ \\
\hline 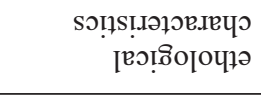 & v & $m$ & $\nabla$ & $\nabla$ & $m$ & N & $n$ & $\nabla$ & $\nabla$ \\
\hline 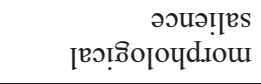 & $N$ & $\nabla$ & $m$ & $m$ & $m$ & $m$ & $n$ & $n$ & $\nabla$ \\
\hline วZỊ ןвว!̣ Кцd & $m$ & $m$ & $m$ & $\nabla$ & $\nabla$ & $n$ & $\nabla$ & $\nabla$ & $\nabla$ \\
\hline $\begin{array}{r}\text { K.єеоินnН } \\
\text { u! әшеน ןвวо }\end{array}$ & 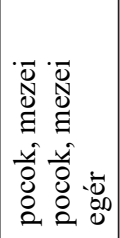 & $\begin{array}{l}: 0 \\
00 \\
0 \\
0 \\
: 0 \\
: 0 \\
>0\end{array}$ & 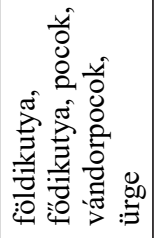 & 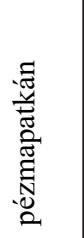 & $\frac{0}{0}$ & 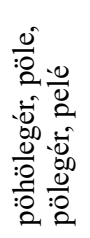 & $\frac{\mathscr{z}}{\stackrel{\Xi}{0}}$ & 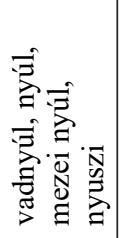 & $\begin{array}{l}\overrightarrow{\tilde{n}} \\
\stackrel{\Xi}{\Xi}\end{array}$ \\
\hline 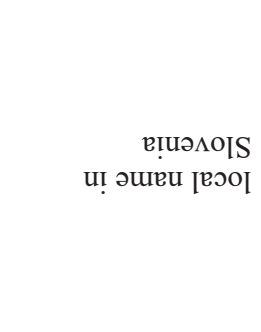 & $\begin{array}{l}\text { 능 } \\
0 \\
0\end{array}$ & 离 & 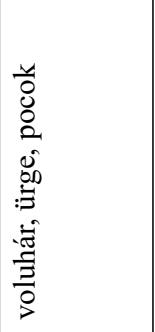 & 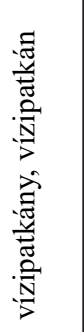 & $\begin{array}{l}0 \\
\frac{0}{0} \\
\frac{10}{1} \\
\vdots 0 \\
\vdots 0 \\
: 0 \\
0 .\end{array}$ & $\begin{array}{l}: 0 \\
: 0 \\
: 0 \\
: 0 \\
: 0 \\
: 0 \\
: 0\end{array}$ & 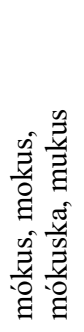 & 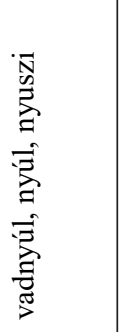 & 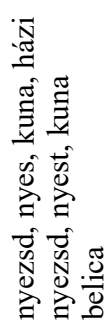 \\
\hline әшนи эџ!̣นวฺ̣s & 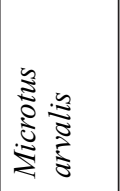 & 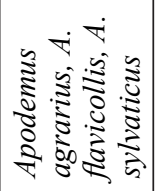 & 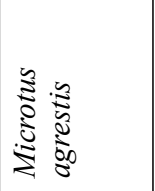 & 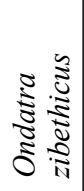 & 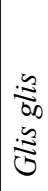 & 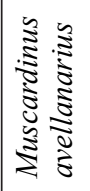 & 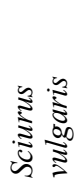 & 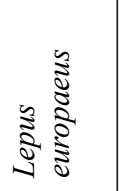 & $\frac{\sqrt{1}}{3}$ \\
\hline & $\stackrel{0}{=}$ & $=$ & $\simeq$ & $\stackrel{n}{-}$ & $\Xi$ & 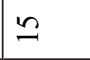 & $\stackrel{0}{-}$ & $\beth$ & $\stackrel{\infty}{-}$ \\
\hline
\end{tabular}




\begin{tabular}{|c|c|c|c|c|c|c|c|c|c|c|c|}
\hline 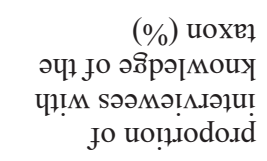 & 官 & ầ & â & â & 导 & 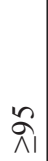 & $\begin{array}{l}\text { Pे } \\
\text { in }\end{array}$ & $\stackrel{n}{\varkappa}$ & $\stackrel{\varkappa}{\Upsilon}$ & $\stackrel{\text { }}{\dddot{\lambda}}$ & \\
\hline 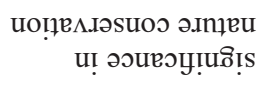 & $\nabla$ & - & 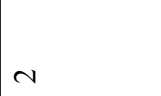 & - & in & 0 & $N$ & 0 & - & - & 8 \\
\hline 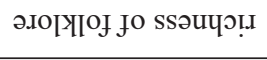 & 0 & N & - & 0 & 0 & $n$ & 一 & $\mathrm{N}$ & $\sim$ & $n$ & $\stackrel{\searrow}{\sim}$ \\
\hline ssəupnjəsn & 0 & - & 0 & 0 & 0 & $\sim$ & 0 & $\nabla$ & $\nabla$ & $\nabla$ & 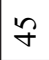 \\
\hline ssəu[nјш.ет & - & $\theta$ & $n$ & $m$ & $\theta$ & in & $n$ & $n$ & $n$ & $\theta$ & $\hat{6}$ \\
\hline sueuny of .əəôuep & 0 & 0 & 0 & - & 0 & $\sim$ & $\sim$ & $n$ & - & N & \\
\hline 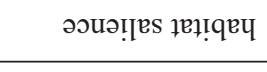 & - & $\theta$ & $\theta$ & n & $m$ & $\nabla$ & $N$ & $m$ & $\nabla$ & $m$ & $\hat{a}$ \\
\hline Кэuənbə.I & $\mathrm{N}$ & $m$ & $m$ & $\nabla$ & $m$ & $n$ & $\sim$ & $n$ & in & $n$ & $\stackrel{8}{-}$ \\
\hline 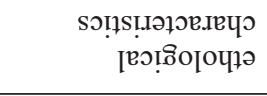 & N & $m$ & in & $\nabla$ & $m$ & $m$ & $\nabla$ & $\nabla$ & in & $\nabla$ & ฮิ \\
\hline 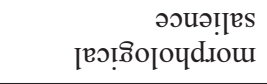 & $\nabla$ & $\nabla$ & $m$ & $n$ & $\nabla$ & $n$ & $\nabla$ & in & $n$ & $n$ & $\stackrel{\varrho}{=}$ \\
\hline 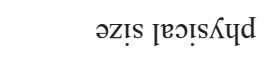 & $\nabla$ & $\nabla$ & & in & in & $n$ & $n$ & $n$ & $n$ & in & $\stackrel{\simeq}{\Xi}$ \\
\hline $\begin{array}{r}\text { KıєôunH } \\
\text { u! әшеu ןвวоI }\end{array}$ & 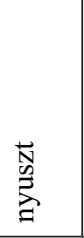 & 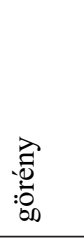 & 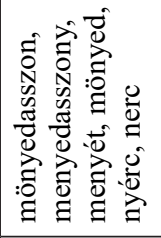 & № & $\frac{\pi}{3}$ & $\frac{\tilde{y}}{0}$ & 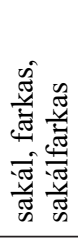 & 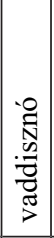 & 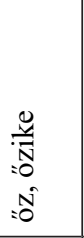 & 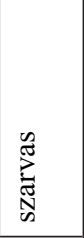 & \\
\hline 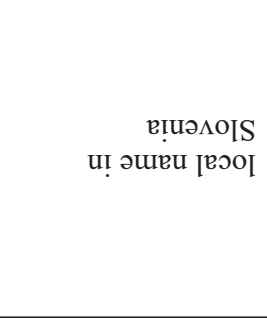 & 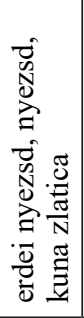 & 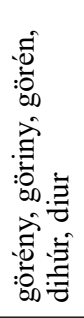 & 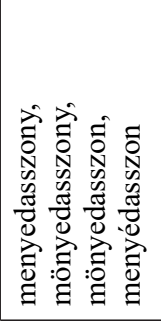 & 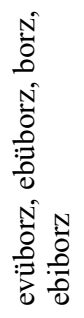 & $\frac{\pi}{5}$ & 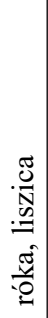 & $\overline{\widetilde{\pi}}$ & 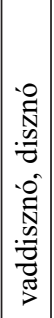 & 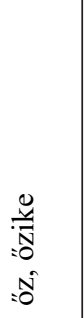 & 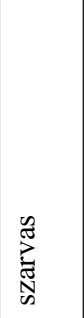 & $\begin{array}{l}\tilde{\Xi} \\
\tilde{\Xi} \\
\tilde{y} \\
\vdots \\
: 0\end{array}$ \\
\hline әшеи әу!̣นәฺฺs & 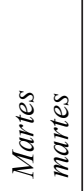 & 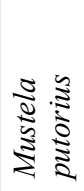 & 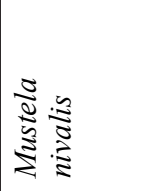 & 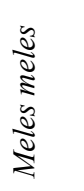 & $\frac{\sqrt{3}}{3}$ & $\begin{array}{c}y \\
\vdots \\
\vdots \\
\vdots \\
\vdots \\
\vdots \\
\vdots \\
\vdots \\
\vdots\end{array}$ & 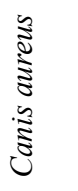 & 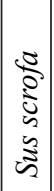 & 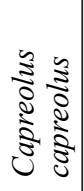 & 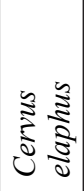 & \\
\hline & $\stackrel{2}{2}$ & $\stackrel{\sim}{~}$ & $\vec{\sim}$ & ป & $\stackrel{\sim}{\sim}$ & $\stackrel{\sim}{\sim}$ & $\stackrel{\sim}{\sim}$ & $\stackrel{\sim}{\sim}$ & $\hat{\sim}$ & $\stackrel{\infty}{\sim}$ & \\
\hline
\end{tabular}




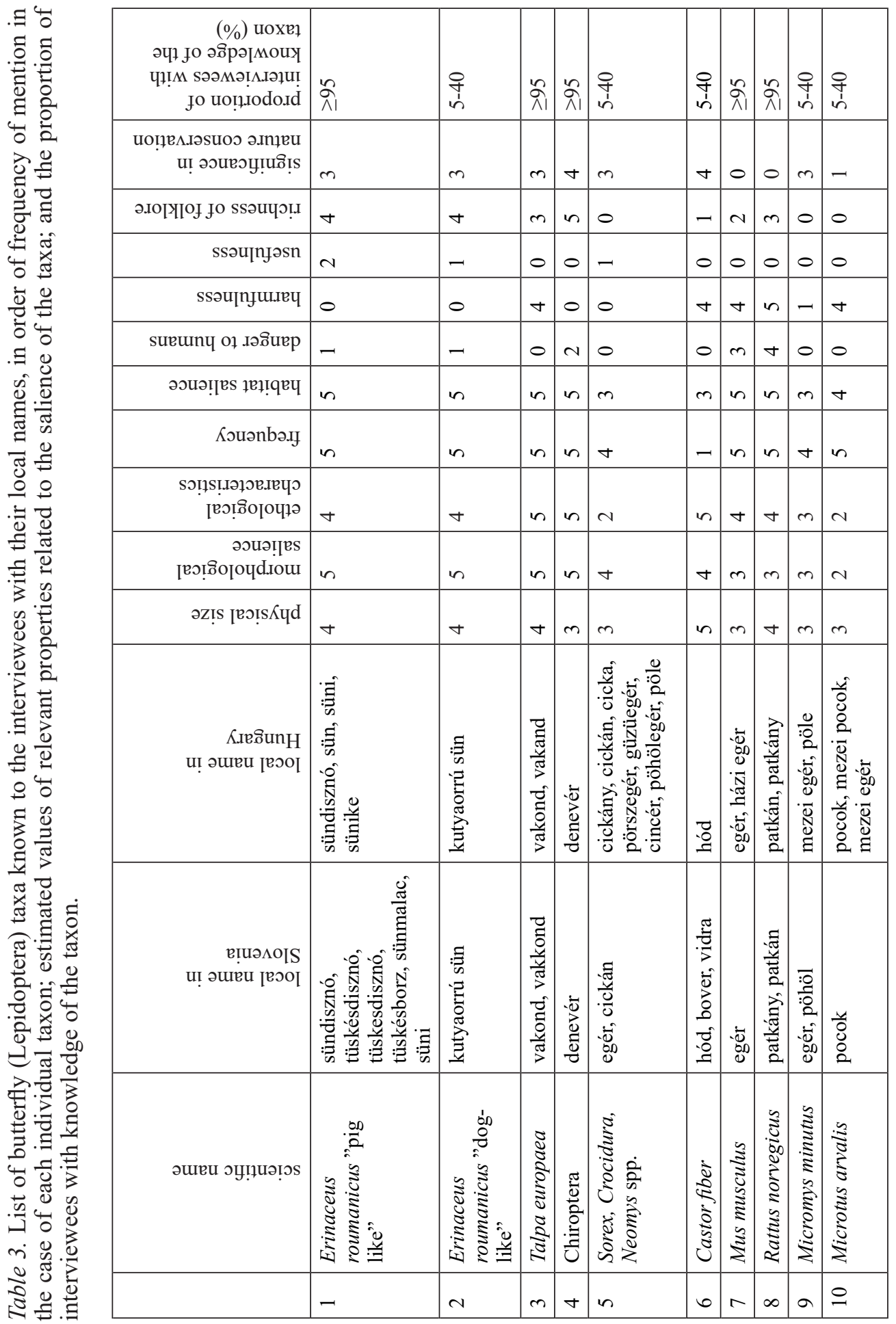




\begin{tabular}{|c|c|c|c|c|c|c|c|c|c|c|}
\hline 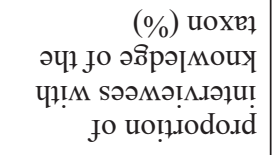 & $\stackrel{n}{v}$ & $\stackrel{n}{\hat{d}}$ & $\mathfrak{v}_{1}$ & $\stackrel{n}{v}$ & $\begin{array}{l}0 \\
0 \\
1 \\
o\end{array}$ & $\stackrel{n}{\hat{n}}$ & $\stackrel{n}{\hat{\Lambda}}$ & â & 吕 & â \\
\hline 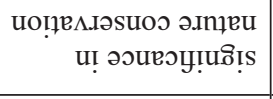 & - & $m$ & 0 & $\forall$ & $\nabla$ & $m$ & $m$ & - & $\nabla$ & - \\
\hline 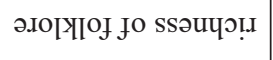 & 0 & 0 & 0 & 0 & 0 & $m$ & N & 0 & 0 & a \\
\hline ssəu[njəsn & 0 & 0 & - & 0 & 0 & 0 & $m$ & 一 & 0 & - \\
\hline ssәuгрушиеч & 0 & $m$ & 0 & $\sim$ & - & - & $m$ & $\nabla$ & - & $\nabla$ \\
\hline sueuny ol .əôuep & 0 & $m$ & 0 & 0 & 0 & 0 & 0 & 0 & 0 & 0 \\
\hline 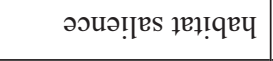 & N & $\nabla$ & $\mathrm{N}$ & N & - & $m$ & $\nabla$ & in & - & $\nabla$ \\
\hline Кэuәnbə.у & $\nabla$ & $n$ & - & $m$ & $m$ & $\nabla$ & $\nabla$ & $\nabla$ & v & $m$ \\
\hline 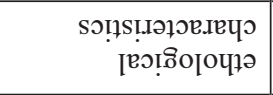 & $m$ & $\nabla$ & $\nabla$ & $m$ & v & $n$ & $\nabla$ & $\nabla$ & v & $m$ \\
\hline 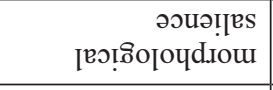 & $\nabla$ & $m$ & $m$ & $m$ & $m$ & $n$ & $n$ & $\nabla$ & $\nabla$ & $\theta$ \\
\hline 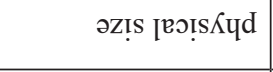 & $m$ & $m$ & $\nabla$ & $\nabla$ & $m$ & $\nabla$ & $\nabla$ & $\nabla$ & $\nabla$ & $\nabla$ \\
\hline 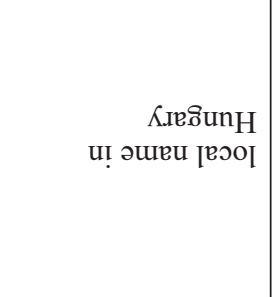 & $\begin{array}{l}\dot{0} \\
0 \\
0 \\
0 \\
0 \\
: 00 \\
0\end{array}$ & 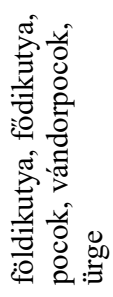 & 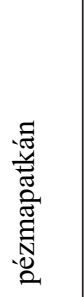 & $\stackrel{0}{0}$ & 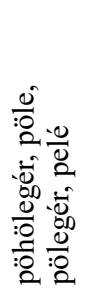 & 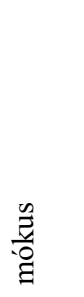 & 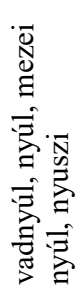 & 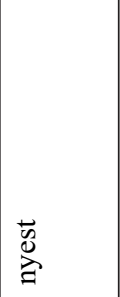 & $\begin{array}{l}\vec{v} \\
0 \\
ٍ \\
ٍ\end{array}$ & 离 \\
\hline 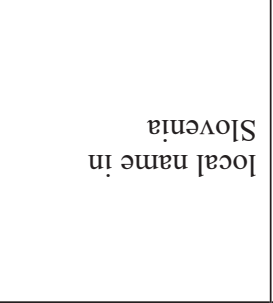 & .্. & 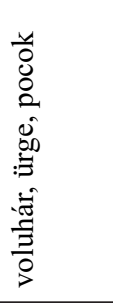 & 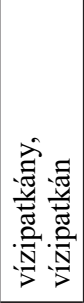 & $\begin{array}{l}0 \\
\frac{0}{0} \\
\frac{1}{1} \\
: 0 \\
\vdots 0 \\
: 0 \\
2\end{array}$ & $\begin{array}{l}: 0 \\
: 0 \\
: 0 \\
2 \\
: 0 \\
: 0 \\
: 0 \\
2\end{array}$ & 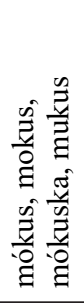 & 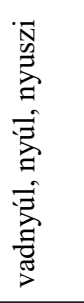 & 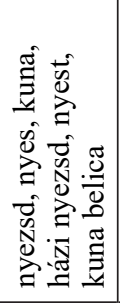 & 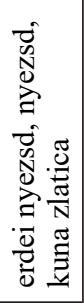 & 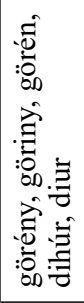 \\
\hline әшеน эџ!ฺนว!ฺs & 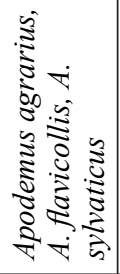 & 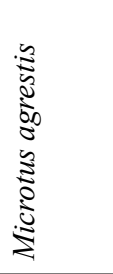 & 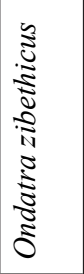 & 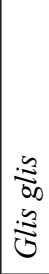 & 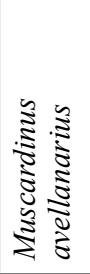 & 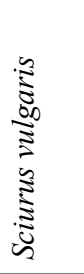 & 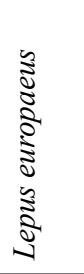 & $\frac{\sqrt{2}}{0}$ & 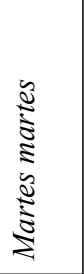 & 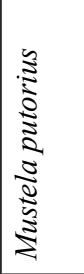 \\
\hline & $=$ & $\simeq$ & 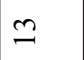 & \pm & 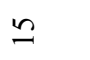 & $\stackrel{0}{0}$ & 드 & $\infty$ & 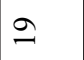 & ิ \\
\hline
\end{tabular}




\begin{tabular}{|c|c|c|c|c|c|c|c|c|c|}
\hline 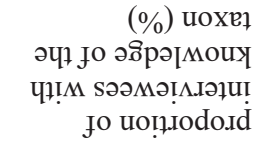 & 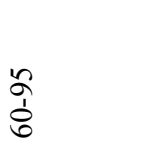 & 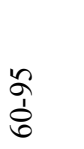 & $\begin{array}{c}\text { ơ } \\
\text { in } \\
\text { ñ }\end{array}$ & 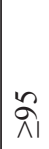 & $\begin{array}{l}\text { P } \\
\text { in } \\
\text { nn }\end{array}$ & $\stackrel{n}{\hat{\Lambda}}$ & $\begin{array}{l}\varkappa \\
\hat{\Lambda}\end{array}$ & 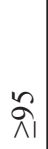 & \\
\hline 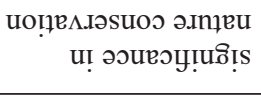 & $\mathrm{N}$ & 一 & $n$ & 0 & $\mathrm{~N}$ & 0 & - & - & 8 \\
\hline 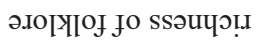 & - & 0 & 0 & $n$ & - & $N$ & $N$ & $n$ & $\stackrel{d}{d}$ \\
\hline ssəu[рృәsn & 0 & 0 & 0 & $a$ & 0 & $\nabla$ & $\nabla$ & $\nabla$ & $\mathscr{\wp}$ \\
\hline ssәu[рушыеч & $m$ & $m$ & $\nabla$ & in & $m$ & $n$ & $m$ & $\nabla$ & $\hat{6}$ \\
\hline suruny ol .əôuep & 0 & - & 0 & 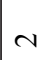 & 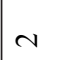 & $n$ & - & $\sim$ & $\hat{\imath}$ \\
\hline 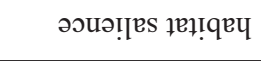 & $\nabla$ & $m$ & $m$ & $\theta$ & $a$ & $m$ & $\nabla$ & $m$ & $\hat{a}$ \\
\hline Кouənbəry & $m$ & $\nabla$ & $m$ & in & $\sim$ & $n$ & $n$ & $n$ & 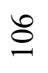 \\
\hline 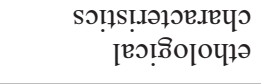 & $n$ & $\nabla$ & $m$ & $m$ & $\theta$ & $\nabla$ & $n$ & $\nabla$ & ชิ \\
\hline 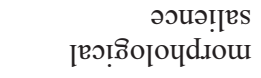 & $m$ & $n$ & $\nabla$ & $n$ & t & $n$ & $n$ & $n$ & $\stackrel{m}{=}$ \\
\hline әZIS ןอว!̣ Кцd & $\nabla$ & n & $n$ & $n$ & in & $n$ & $n$ & $n$ & $\stackrel{\cong}{\Xi}$ \\
\hline 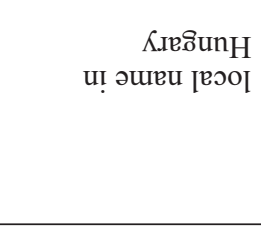 & 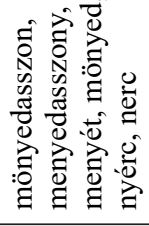 & Nõ & $\frac{\pi}{\frac{\pi}{7}}$ & $\frac{\mathscr{g}}{0}$ & 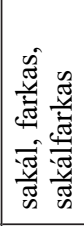 & 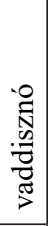 & 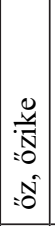 & 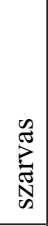 & \\
\hline 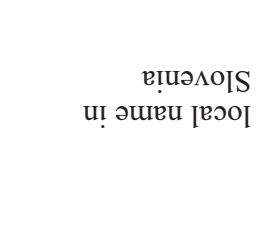 & 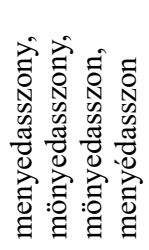 & 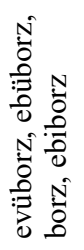 & $\frac{\pi}{\frac{\pi}{5}}$ & 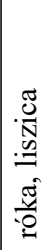 & 茎 & 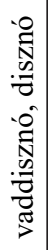 & 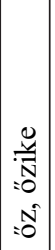 & 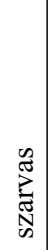 & 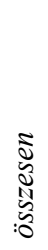 \\
\hline әயвน әழฺ̣யวழฺs & 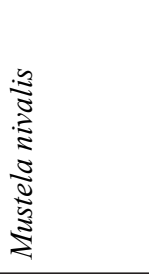 & 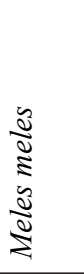 & 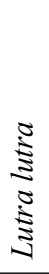 & \begin{tabular}{|c}
2 \\
$\vdots$ \\
$\vdots$ \\
$\vdots$ \\
$\vdots$ \\
$\vdots$ \\
$\vdots$ \\
$\vdots$ \\
$\vdots$
\end{tabular} & 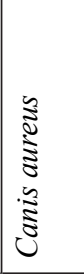 & $\begin{array}{c}0 \\
0 \\
0 \\
5 \\
5 \\
\vdots \\
5 \\
5\end{array}$ & 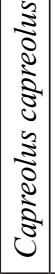 & 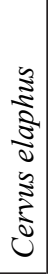 & \\
\hline & $\vec{\sim}$ & ป & $\tilde{\imath}$ & $\stackrel{4}{4}$ & 2 & $\stackrel{\sim}{\sim}$ & $\hat{\sim}$ & $\stackrel{\sim}{\sim}$ & \\
\hline
\end{tabular}


Table 4. List of the invertebrate taxa (excluding butterflies) known to the interviewees, with their local names, given in the order of frequency with which they were mentioned in the case of each individual taxon. (* - correctly known taxon, but not called by its name in the given region; \% - proportion of interviewees with knowledge of the taxon)

\begin{tabular}{|c|c|c|c|c|}
\hline & scientific name & local name in Slovenia & local name in Hungary & $\%$ \\
\hline & Myriapoda & & & \\
\hline 1 & $\begin{array}{l}\text { Julidae e.g. Megaphyllum } \\
\text { unilineatum }\end{array}$ & $*$ & $*$ & $\leq 5$ \\
\hline \multirow[t]{2}{*}{2} & $\begin{array}{l}\text { Lithobius spp. e.g. Lithobius } \\
\text { forficatus }\end{array}$ & százlábú, sztonoga & százlábú & $5-40$ \\
\hline & Arachnida & & & \\
\hline 1 & $\begin{array}{l}\text { Ixodes spp. e.g. Ixodes } \\
\text { ricinus }\end{array}$ & kullancs, kulláncs & kullancs, kulláncs & $\geq 95$ \\
\hline 2 & Tetranychus urticae & - & $*$ & $\leq 5$ \\
\hline 3 & Varroa destructor & - & atka, méhatka & $\leq 5$ \\
\hline 4 & $\begin{array}{l}\text { Araneae e.g. Tegenaria } \\
\text { domestica }\end{array}$ & pók & pók & $\geq 95$ \\
\hline 5 & Misumena vatia & mérgespók & - & $\leq 5$ \\
\hline 6 & $\begin{array}{l}\text { Araneus spp. e.g. Araneus } \\
\text { diadematus }\end{array}$ & keresztespók & keresztespók & $5-40$ \\
\hline \multirow[t]{2}{*}{7} & $\begin{array}{l}\text { Pholcidae e.g. Holocnemus } \\
\text { pluchei, Opiliones e.g. } \\
\text { Phalangium opilio }\end{array}$ & pók & kaszáspók & $40-60$ \\
\hline & Crustacea & & & \\
\hline 1 & Astacus astacus & rák & rák & $\geq 95$ \\
\hline \multirow[t]{2}{*}{2} & $\begin{array}{l}\text { Oniscidea e.g. Armadillidium } \\
\text { vulgare }\end{array}$ & $*$ & pincebogár & $5-40$ \\
\hline & Orthoptera & & & \\
\hline 1 & Tettigonia viridissima & $\begin{array}{l}\text { sáska, kaszás, ződ } \\
\text { kaszás }\end{array}$ & sáska, kaszás & $60-95$ \\
\hline 2 & Oecanthus pellucens & csürbogár & - & $\leq 5$ \\
\hline 3 & $\begin{array}{l}\text { Caelifera e.g. Calliptamus } \\
\text { italicus }\end{array}$ & szecsku, szöcske & szöcske, szecsku, sáska & $40-60$ \\
\hline 4 & Gryllus campestris & tücsök, cslicsek & tücsök, pücsök & $\geq 95$ \\
\hline \multirow[t]{2}{*}{5} & Gryllotalpa gryllotalpa & lóbogár, lótetü, lútetü & lótetü & $60-95$ \\
\hline & Hemiptera & & & \\
\hline 1 & $\begin{array}{l}\text { Gerris spp. e.g. Gerris } \\
\text { paludum }\end{array}$ & víziszöcske & $\begin{array}{l}\text { vizipók, víziborjú (sic!), } \\
\text { pók }\end{array}$ & $5-40$ \\
\hline 2 & Pyrrhocoris apterus & $*$ & $\begin{array}{l}\text { bodobácsbogár, } \\
\text { baszóbogár, bodobács }\end{array}$ & $60-95$ \\
\hline 3 & Eurydema ornata & - & káposztapoloska & $\leq 5$ \\
\hline
\end{tabular}




\begin{tabular}{|c|c|c|c|c|}
\hline & scientific name & local name in Slovenia & local name in Hungary & $\%$ \\
\hline 4 & $\begin{array}{l}\text { Dolycoris baccarum and } \\
\text { similar species }\end{array}$ & $\begin{array}{l}\text { büdösbákó, büdösbáku, } \\
\text { büdöske }\end{array}$ & $\begin{array}{l}\text { büdösmargit, } \\
\text { margitbogár, büdösbogár, } \\
\text { kalamászbogár, marinka, } \\
\text { büdösbence, büdösmátyás }\end{array}$ & $\geq 95$ \\
\hline 5 & $\begin{array}{l}\text { Cercopidae e.g. Philaenus } \\
\text { spumarius }\end{array}$ & hab, ökörnyál & hab & $60-95$ \\
\hline \multirow[t]{2}{*}{6} & Cimex lectularius & poloska & - & $60-95$ \\
\hline & Coleoptera & & & \\
\hline 1 & $\begin{array}{l}\text { Carabidae e.g. Zabrus } \\
\text { tenebrioides }\end{array}$ & géber & géber, bogár & $5-40$ \\
\hline 2 & $\begin{array}{l}\text { Geotrupes spp. e.g. } \\
\text { Geotrupes vernalis }\end{array}$ & galacsinhajtó & $\begin{array}{l}\text { ganajtúró bogár, szartúró } \\
\text { bogár }\end{array}$ & $60-95$ \\
\hline 3 & Leptinotarsa decemlineata & krumplibogár & $\begin{array}{l}\text { krumplibogár, korodádó, } \\
\text { kororádóbogár }\end{array}$ & $\geq 95$ \\
\hline 4 & $\begin{array}{l}\text { Chaetocnema spp., } \\
\text { Phyllotrema spp. e.g. } \\
\text { Chaetocnema tibialis }\end{array}$ & - & balha, káposztabolha & $5-40$ \\
\hline 5 & Tenebrio molitor & - & lisztféreg & $\leq 5$ \\
\hline 6 & $\begin{array}{l}\text { Curculionidae e.g. Larinus } \\
\text { turbinatus }\end{array}$ & - & $*$ & $\leq 5$ \\
\hline 7 & Ceutorhynchus macula-alba & - & bogár & $\leq 5$ \\
\hline 8 & Sitophilus granarius & zsizsik & zsizsik, gabonazsizsik & $5-40$ \\
\hline 9 & $\begin{array}{l}\text { Bruchus pisorum, } \\
\text { Acanthoscelides obtectus }\end{array}$ & zsizsik & zsizsik & $5-40$ \\
\hline 10 & Lytta vesicatoria & kőrisbogár, büdösbogár & kőrisbogár & $5-40$ \\
\hline 11 & Melolontha melolontha & $\begin{array}{l}\text { cserebogár, csimmasz, } \\
\text { csimmaz }\end{array}$ & cserebogár, csimmasz & $\geq 95$ \\
\hline 12 & $\begin{array}{l}\text { Cerambyx cerdo and similar } \\
\text { species }\end{array}$ & - & cincér, hőscincér, facincér & $5-40$ \\
\hline 13 & Lucanus cervus & istenökre, szarvasbogár & szarvasbogár, istenökre & $\geq 95$ \\
\hline 14 & $\begin{array}{l}\text { Coccinella septempunctata, } \\
\text { C. magnifica }\end{array}$ & bödebence, katicabogár & $\begin{array}{l}\text { bödebence, katicabogár, } \\
\text { katica, rendes katica }\end{array}$ & $\geq 95$ \\
\hline 15 & Harmoia axyridis & $\begin{array}{l}\text { bödebence, katicabogár, } \\
\text { bödebogár }\end{array}$ & bödebence & $40-60$ \\
\hline 16 & $\begin{array}{l}\text { Psyllobora } \\
\text { vigintiduopunctata }\end{array}$ & - & sárga katicabogár & $\leq 5$ \\
\hline 17 & Lymexylon navale & - & hajófúró bogár & $\leq 5$ \\
\hline 18 & $\begin{array}{l}\text { Cetonia aurata and Protaetia } \\
\text { e.g. Protaetia affinis }\end{array}$ & szentjánosbogár & $\begin{array}{l}\text { szentjánosbogár, } \\
\text { rózsabogár }\end{array}$ & $60-95$ \\
\hline
\end{tabular}




\begin{tabular}{|c|c|c|c|c|}
\hline & scientific name & local name in Slovenia & local name in Hungary & $\%$ \\
\hline 19 & $\begin{array}{l}\text { Lampyris noctiluca, } \\
\text { Lamprohiza splendidula }\end{array}$ & $\begin{array}{l}\text { Szentjánosbogár, } \\
\text { szentivánbogár, } \\
\text { ivánbogár }\end{array}$ & szentjánosbogár & $\geq 95$ \\
\hline 20 & Ips spp. e.g. Ips typographus & szú & szu, szú, faszú & $60-95$ \\
\hline 21 & Anobium punctatum & - & faszú, szú & $\leq 5$ \\
\hline 22 & Blaps spp. e.g. Blaps lethifera & $\begin{array}{l}\text { büdösbogár, } \\
\text { keserübogár }\end{array}$ & büdösbogár, pincebogár & $5-40$ \\
\hline \multirow[t]{2}{*}{23} & $\begin{array}{l}\text { Agriotes spp. e.g. Agriotes } \\
\text { sputator }\end{array}$ & drótféreg, szárazféreg & drótféreg & $5-40$ \\
\hline & Hymenoptera & & & \\
\hline 1 & Apis mellifera & méhe, méhecske, méh & $\begin{array}{l}\text { méh, méhe, háziméhe, } \\
\text { méhi }\end{array}$ & $\geq 95$ \\
\hline 2 & Apis mellifera var. ligustica & - & vadméhe & $5-40$ \\
\hline 3 & $\begin{array}{l}\text { Andrenidae, Colletidae, } \\
\text { Melittidae, Halictidae, } \\
\text { Megachilidae }\end{array}$ & földiméhe & földiméhe, földiméhi & $5-40$ \\
\hline 4 & $\begin{array}{l}\text { Halictidae e.g. Halictus } \\
\text { sexcinctus }\end{array}$ & - & $\begin{array}{l}\text { vadméhe, méh, méhe, } \\
\text { földiméhe }\end{array}$ & $\leq 5$ \\
\hline 5 & $\begin{array}{l}\text { Bombus terrestris and similar } \\
\text { species }\end{array}$ & $\begin{array}{l}\text { dongó, földiméhe, } \\
\text { födiméhe, földiméh }\end{array}$ & $\begin{array}{l}\text { födiméhe, poszméh, } \\
\text { földiméhi }\end{array}$ & $60-95$ \\
\hline 6 & $\begin{array}{l}\text { Tetramorium caespitum and } \\
\text { similar species }\end{array}$ & hangya & hangya & $\geq 95$ \\
\hline 7 & Formica rufa & vöröshangya & hangya & $5-40$ \\
\hline 8 & $\begin{array}{l}\text { ants from different species } \\
\text { and casts with wings }\end{array}$ & szárnyashangya & szárnyashangya & $60-95$ \\
\hline 9 & $\begin{array}{l}\text { Camponotus spp. e.g. } \\
\text { Camponotus ligniperda }\end{array}$ & nagy fekete hangya & nagy fekete hangya & $60-95$ \\
\hline 10 & Lasius flavus, L. umbratus & vöröshangya & vöröshangya & $60-95$ \\
\hline 11 & Sceliphron destillatorium & szakandék & - & $\leq 5$ \\
\hline 12 & Vespa crabro & darázs, lódarázs & $\begin{array}{l}\text { lódarázs, darázs, } \\
\text { szakadék, méhfarkas }\end{array}$ & $\geq 95$ \\
\hline 13 & $\begin{array}{l}\text { Vespula vulgaris, } \\
\text { Paravespula germanica }\end{array}$ & szakandik, szakandék & darázs, szakadék & $\geq 95$ \\
\hline 14 & Polistes gallicus & szakandik, szakandék & kecskedarázs, szakadék & $\leq 5$ \\
\hline 15 & Cynips quercusfolii & gubacs, gubola & - & $5-40$ \\
\hline 16 & Rhodites rosae & $*$ & $*$ & $5-40$ \\
\hline \multirow[t]{2}{*}{17} & Andricus hungaricus & $*$ & gubacs, guba & $60-95$ \\
\hline & Diptera & & & \\
\hline 1 & $\begin{array}{l}\text { Aedes spp. and similar } \\
\text { species }\end{array}$ & szúnyog, szunyog & szunyog & $\geq 95$ \\
\hline
\end{tabular}




\begin{tabular}{|c|c|c|c|c|}
\hline & scientific name & local name in Slovenia & local name in Hungary & $\%$ \\
\hline 2 & $\begin{array}{l}\text { Tipula spp. e.g. Tipula } \\
\text { maxima }\end{array}$ & szunyog & $\begin{array}{l}\text { szunyog, vacsoravesztő, } \\
\text { szitakötő }\end{array}$ & $60-95$ \\
\hline 3 & $\begin{array}{l}\text { Ceratopogonidae e.g. } \\
\text { Culicoides imicola }\end{array}$ & muslinca, muszlinca & $*$ & $5-40$ \\
\hline 4 & $\begin{array}{l}\text { Drosophila spp. e.g. } \\
\text { Drosophila melanogaster }\end{array}$ & $\begin{array}{l}\text { muslinca, muslica, } \\
\text { muszlinca }\end{array}$ & muszlinca & $\geq 95$ \\
\hline 5 & $\begin{array}{l}\text { Psychodidae e.g. Clogmia } \\
\text { albipunctata }\end{array}$ & lepke & - & $\leq 5$ \\
\hline 6 & Braula coeca & baroza & - & $\leq 5$ \\
\hline 7 & $\begin{array}{l}\text { Haematopota spp. e.g. } \\
\text { Haematopota pluvialis }\end{array}$ & dongó & katonadongó & $60-95$ \\
\hline 8 & Tabanus bovinus & dongó, lódongó & dongó, bögöly & $\geq 95$ \\
\hline 9 & Hypoderma bovis & - & féreg, bödölle, gödölle & $\leq 5$ \\
\hline 10 & Hypoderma diana & $*$ & bödölle, gödölle & $5-40$ \\
\hline 11 & Asilidae & légy & légy & $\leq 5$ \\
\hline 12 & Musca domestica & légy, házilégy & bogár, rendes légy & $\geq 95$ \\
\hline 13 & Haematobia irritans & - & légy & $5-40$ \\
\hline 14 & Sarcophaga carnaria & féregszaró, légy & húsbogár, dögbogár & $60-95$ \\
\hline 15 & Calliphora vicina & kék légy & húsbogár & $\leq 5$ \\
\hline 16 & $\begin{array}{l}\text { Lucilia spp. e.g. Lucilia } \\
\text { caesar }\end{array}$ & $\begin{array}{l}\text { zöld légy, mészároslégy, } \\
\text { légy }\end{array}$ & légy, dögbogár, húsbogár & $60-95$ \\
\hline 17 & $\begin{array}{l}\text { Chrysops spp. e.g. Chrysops } \\
\text { caecutiens }\end{array}$ & dongó & $\begin{array}{l}\text { tarkaszárnyú bögöly, tarka } \\
\text { dongó }\end{array}$ & $5-40$ \\
\hline 18 & Gasterophilus intestinalis & $*$ & - & $\leq 5$ \\
\hline 19 & $\begin{array}{l}\text { Syrphidae e.g. Syrphus } \\
\text { ribesii }\end{array}$ & légy & - & $\leq 5$ \\
\hline 20 & Hippobosca longipennis & kutyalégy, kutyabogár & lókulláncs & $5-40$ \\
\hline \multirow[t]{2}{*}{21} & Rhagoletis cerasi s.l. & - & féreg & $60-95$ \\
\hline & Insecta & & & \\
\hline 1 & Aphididae e.g. Apis pomi & $*$ & tetü, levéltetü, rózsatetü & $5-40$ \\
\hline 2 & Myzus cerasi & penészbogár & tetü, fekete tetü & $5-40$ \\
\hline 3 & $\begin{array}{l}\text { Aleyrodina e.g. Aleyrodes } \\
\text { proletella }\end{array}$ & - & levéltetü & $5-40$ \\
\hline 4 & $\begin{array}{l}\text { Coccoidea e.g. Aspidiotus } \\
\text { nerii }\end{array}$ & - & atka & $\leq 5$ \\
\hline 5 & Menacanthus stramineus & $\begin{array}{l}\text { tyuktetü, tiktetü, tetü, } \\
\text { bolha }\end{array}$ & tiktetü, tyuktetü & $60-95$ \\
\hline 6 & Haematopinus suis & tetü, disznótetü & - & $5-40$ \\
\hline 7 & Bovicola bovis & - & tehéntetü & $\leq 5$ \\
\hline
\end{tabular}




\begin{tabular}{|c|c|c|c|c|}
\hline & scientific name & local name in Slovenia & local name in Hungary & $\%$ \\
\hline 8 & Pediculus humanus capitis & tetü, fejtetü & tetü, fejtetü & $\geq 95$ \\
\hline 9 & Pediculus humanus humanus & tetü, ruhatetü & tetü & $60-95$ \\
\hline 10 & Pulex irritans & bolha, bóha & bolha, balha & $\geq 95$ \\
\hline 11 & Ctenocephalides canis & bolha, kutyabolha & balha, bolha & $5-40$ \\
\hline 12 & Mantis religiosa & $\begin{array}{l}\text { imádkozó sáska, } \\
\text { bogomolka }\end{array}$ & imádkozó sáska, sáska & $5-40$ \\
\hline 13 & Blatta orientalis & csótány & géber & $5-40$ \\
\hline 14 & $\begin{array}{l}\text { Dermaptera e.g. Forficula } \\
\text { auricularia }\end{array}$ & fülbemászó, fülmászó & fülmászó, fülbemászó & $60-95$ \\
\hline 15 & $\begin{array}{l}\text { Ephemeroptera e.g. } \\
\text { Ephemera danica }\end{array}$ & - & kérész & $\leq 5$ \\
\hline \multirow[t]{2}{*}{16} & Odonata e.g. Anax imperator & $\begin{array}{l}\text { kigyupásztor, } \\
\text { kigyópásztor, szitakötő }\end{array}$ & szitakötő, kigyupásztor & $\geq 95$ \\
\hline & Nematoda & & & \\
\hline 1 & Toxocara canis & giliszta & - & $5-40$ \\
\hline 2 & Toxocara cati, T. leonina & geleszta, giliszta & - & $\leq 5$ \\
\hline 3 & Ascaris suum & - & orsós, giliszta, orsóféreg & $5-40$ \\
\hline 4 & Dictyocaulus viviparus & - & tüdőféreg, féreg & $\leq 5$ \\
\hline \multirow[t]{2}{*}{5} & Eisenia fetida & - & giliszta & $5-40$ \\
\hline & Platyhelminthes & & & \\
\hline 1 & $\begin{array}{l}\text { Taenia solium, } \\
\text { Taeniarhynchus saginatus }\end{array}$ & geleszta, pántlikgiliszta & giliszta & $5-40$ \\
\hline \multirow[t]{2}{*}{2} & Fasciola hepatica & májmétel, májlepke & - & $40-60$ \\
\hline & Annelida & & & \\
\hline 1 & $\begin{array}{l}\text { Lumbricus spp. e.g. } \\
\text { Lumbricus terrestris }\end{array}$ & $\begin{array}{l}\text { giliszta, földigiliszta, } \\
\text { brázdonbillegető }\end{array}$ & $\begin{array}{l}\text { barázdaféreg, brázdaféreg, } \\
\text { giliszta }\end{array}$ & $\geq 95$ \\
\hline \multirow[t]{2}{*}{2} & $\begin{array}{l}\text { Hirudo officinalis, Hirudo } \\
\text { verbana }\end{array}$ & pióca, piuca & pióca & $60-95$ \\
\hline & Mollusca & & & \\
\hline 1 & $\begin{array}{l}\text { Arion, Limax spp. e.g. Limax } \\
\text { maximus }\end{array}$ & $\begin{array}{l}\text { kágyu, kágyus csiga, } \\
\text { csigakágyu, meztelen } \\
\text { csiga }\end{array}$ & $\begin{array}{l}\text { kágyú, kágyillu, meztelen } \\
\text { csiga, köpeszcsiga }\end{array}$ & $60-95$ \\
\hline 2 & Arion lusitanicus & $\begin{array}{l}\text { vörös kágyú, vörös } \\
\text { csiga, kágyú }\end{array}$ & $\begin{array}{l}\text { kágyú, kágyillu, vörös } \\
\text { csiga, vörös kágyú, } \\
\text { köpeszcsiga }\end{array}$ & $\geq 95$ \\
\hline 3 & Agriolimax agrestis & fehér kágyu & - & $\leq 5$ \\
\hline 4 & $\begin{array}{l}\text { Gastropoda e.g. Helix } \\
\text { pomatia }\end{array}$ & csiga & csiga & $\geq 95$ \\
\hline 5 & Xerolenta obvia & csiga & csiga & $5-40$ \\
\hline 6 & $\begin{array}{l}\text { Bivalvia e.g. Anodonta } \\
\text { cygnea }\end{array}$ & kagyló & kagyló & $60-95$ \\
\hline
\end{tabular}


Table 5. List of fish, amphibian, and reptile taxa known by the interviewees with their local names, given in the order of frequency with which they were mentioned in the case of each individual taxon ( $\%$ - proportion of interviewees with a knowledge of the taxon)

\begin{tabular}{|c|c|c|c|c|}
\hline & scientific name & local name in Slovenia & local name in Hungary & $\%$ \\
\hline & Osteichthyes & & & \\
\hline 1 & Salmo trutta & pisztráng & pisztráng & $5-40$ \\
\hline 2 & Esox lucius & csuka & csuka & $5-40$ \\
\hline 3 & Cyprinus carpio & ponty & ponty & $\begin{array}{l}60- \\
95\end{array}$ \\
\hline 4 & $\begin{array}{l}\text { Scardinius erythrophthalmus } \\
\text { and similar species }\end{array}$ & keszeg & - & $5-40$ \\
\hline 5 & Squalius cephalus & domonkó, pénzes & pénzes & $5-40$ \\
\hline 6 & Ctenopharyngodon idella & amúr & - & $\leq 5$ \\
\hline 7 & $\begin{array}{l}\text { Barbatula barbatula, } \\
\text { Cobitis elongatoides }\end{array}$ & csik, picsarági & - & $5-40$ \\
\hline \multirow[t]{2}{*}{8} & Lota lota & menyhal & - & $\leq 5$ \\
\hline & Amphibia & & & \\
\hline 1 & $\begin{array}{l}\begin{array}{l}\text { Triturus spp., Lissottriton } \\
\text { vulgaris }\end{array} \\
\end{array}$ & $\begin{array}{l}\text { vízibornyú, gyik, } \\
\text { mocsarád, gőte }\end{array}$ & göte, víziborjú & $5-40$ \\
\hline 2 & Salamandra salamandra & mocsarád, mocserád & $\begin{array}{l}\text { göte, fodros szalamandra, } \\
\text { alpesi szalamandra }\end{array}$ & $5-40$ \\
\hline 3 & $\begin{array}{l}\text { Bombina bombina, } B . \\
\text { variegata }\end{array}$ & béka & béka, unkabéka, unka & $5-40$ \\
\hline 4 & Bufotes viridis (Bufo bufo) & $\begin{array}{l}\text { taracskosbéka, } \\
\text { varangyosbéka, } \\
\text { boszorkánybéka, } \\
\text { bukszabéka, króta }\end{array}$ & $\begin{array}{l}\text { varangyosbéka, taracskos } \\
\text { béka, bukszabéka, } \\
\text { varangy, torubeli } \\
\text { béka, targyakos béka, } \\
\text { katonabéka }\end{array}$ & $\geq 95$ \\
\hline 5 & Bufo bufo & - & varangy & $5-40$ \\
\hline 6 & Hyla arborea & $\begin{array}{l}\text { levelibéka, béka, } \\
\text { zöldlevelü béka, regica, } \\
\text { regice }\end{array}$ & levelibéka, levelesbéka & $\geq 95$ \\
\hline 7 & Rana dalmatina & hugyosbéka & $\begin{array}{l}\text { hugyosbéka, katonabéka, } \\
\text { bubosbéka }\end{array}$ & $\begin{array}{l}60- \\
95\end{array}$ \\
\hline 8 & Rana arvalis & kék béka & - & $\leq 5$ \\
\hline \multirow[t]{2}{*}{9} & Pelophylax spp. & - & béka, kecskebéka & $5-40$ \\
\hline & Reptilia & & & \\
\hline 1 & Emys orbicularis & teknyősbéka, teknős & teknősbéka, teknős, teknőc & $\begin{array}{l}60- \\
95\end{array}$ \\
\hline 2 & Lacerta agilis & $\begin{array}{l}\text { gyík, martincsek, barna } \\
\text { gyík }\end{array}$ & gyik, gyík, szürkegyik & $\geq 95$ \\
\hline
\end{tabular}




\begin{tabular}{|c|c|c|c|c|}
\hline & scientific name & local name in Slovenia & local name in Hungary & $\%$ \\
\hline 3 & Lacerta viridis & zöld gyik, gyik, gyík & zöld gyik & $\begin{array}{l}60- \\
95\end{array}$ \\
\hline 4 & Anguis fragilis & vakkigyu & $\begin{array}{l}\text { vakkigyó, rézsikló, } \\
\text { lábatlan gyík, kuszma, } \\
\text { törékenygyík }\end{array}$ & $\geq 95$ \\
\hline 5 & Natrix natrix & $\begin{array}{l}\text { kigyu, kígyó, sikló, } \\
\text { vízisikló, belúska, fehér } \\
\text { fülü, csipükigyó }\end{array}$ & sikló, kígyó, vízisikló & $\geq 95$ \\
\hline 6 & Vipera berus & $\begin{array}{l}\text { mérgeskígyó, vipera, } \\
\text { homoki vipera, gád, } \\
\text { virágos kigyu, csipükigyu }\end{array}$ & $\begin{array}{l}\text { kigyu, kígyó, } \\
\text { mérgeskígyó, vipera, } \\
\text { keresztes vipera }\end{array}$ & $\begin{array}{l}60- \\
95\end{array}$ \\
\hline 7 & "house snake" & kígyó & kígyó & $\begin{array}{l}60- \\
95\end{array}$ \\
\hline
\end{tabular}

Table 6. List of bird taxa known by the interviewees with their local names, given in the order of frequency with which they were mentioned in the case of each individual taxon ( $\%$ - proportion of interviewees with a knowledge of the taxon)

\begin{tabular}{|l|l|l|l|l|}
\hline & scientific name & local name in Slovenia & local name in Hungary & $\%$ \\
\hline 1 & Ardea cinerea & $\begin{array}{l}\text { szürkegém, vasgém, } \\
\text { szürke vasgém }\end{array}$ & gém, szürkegém & $5-40$ \\
\hline 2 & Ardea alba & fehér vasgém & - & $\leq 5$ \\
\hline 3 & Ciconia ciconia & gólya & gólya & $\geq 95$ \\
\hline 4 & Ciconia nigra & feketegém & fekete gólya & $5-40$ \\
\hline 5 & Anas platyrhynchos & vadkacsa, vadréce, kacsa & vadkacsa & $5-40$ \\
\hline 6 & Anser anser & - & szürkelúd & $\leq 5$ \\
\hline 7 & Accipiter gentilis & kánya, igazi kánya & kánya, vércse, héja & $60-95$ \\
\hline 8 & Buteo buteo & $\begin{array}{l}\text { nyulászkánya, } \\
\text { nyulászókánya }\end{array}$ & $\begin{array}{l}\text { nyulászó kánya, } \\
\text { egerészölyv, kánya, ölyv, }\end{array}$ & $5-40$ \\
\hline 9 & Falco tinnunculus sas & vércse & $5-40$ \\
\hline 10 & Phasianus colchicus & vércse & fácán & $\geq 95$ \\
\hline 11 & Perdix perdix & fogoly, fácán & fogoly, fogolymadár & $60-95$ \\
\hline 12 & Coturnix coturnix & pitypalaty, fürj & fürj, pitypalaty & $40-60$ \\
\hline 13 & Tetrao urogallus & vadkakas & fajd, fajdkakas & $5-40$ \\
\hline 14 & Crex crex & - & haris & $\leq 5$ \\
\hline 15 & Streptopelia turtur & vadgalamb & vadgalamb & $5-40$ \\
\hline 16 & Streptopelia decaocto & gelice, gerlice, vadgerlice & gerlice, galamb & $\geq 95$ \\
\hline 17 & Columba palumbus & $\begin{array}{l}\text { vadgalamb, pudpudum } \\
\text { szidi }\end{array}$ & vadgalamb & $5-40$ \\
\hline & & & & \\
\hline
\end{tabular}




\begin{tabular}{|c|c|c|c|c|}
\hline & scientific name & local name in Slovenia & local name in Hungary & $\%$ \\
\hline 18 & Asio otus & $\begin{array}{l}\text { bagoly, bagó, } \\
\text { szürkebagoly, fülesbagoly, } \\
\text { nagyorrú bagó, }\end{array}$ & $\begin{array}{l}\text { bagoly, fülesbagoly, huhu, } \\
\text { uhu }\end{array}$ & $\geq 95$ \\
\hline 19 & Athene noctua & halálbagoly & halálmadár & $5-40$ \\
\hline 20 & Tyto alba & - & hóbagoly & $\leq 5$ \\
\hline 21 & Scolopax rusticola & szalonka, szloka, szlokom & szalonka & $40-60$ \\
\hline 22 & Vanellus vanellus & bibic & - & $\leq 5$ \\
\hline 23 & Cuculus canorus & $\begin{array}{l}\text { kukukk, kakukk, } \\
\text { kakukkmadár }\end{array}$ & kakukk & $\geq 95$ \\
\hline 24 & Upupa epops & $\begin{array}{l}\text { huputa, bubosbanka, } \\
\text { upkás, hupkas, } \\
\text { búbosblanka }\end{array}$ & $\begin{array}{l}\text { büdös huputár, } \\
\text { bubosbanka }\end{array}$ & $60-95$ \\
\hline 25 & Coracias garrulus & $\begin{array}{l}\text { zödbákán, kalakótya, } \\
\text { zöldbákány }\end{array}$ & zöldbákán & $5-40$ \\
\hline 26 & Dendrocopus spp. & harkály & harkály & $60-95$ \\
\hline 27 & Dryocopus martius & fekete küllő & - & $\leq 5$ \\
\hline 28 & Picus viridis & küllö & - & $\leq 5$ \\
\hline 29 & Alauda arvensis & pacsirta & pacsirta & $5-40$ \\
\hline 30 & Galerida cristata & $\begin{array}{l}\text { pityér, barázdabillegető } \\
\text { (sic!) }\end{array}$ & - & $5-40$ \\
\hline 31 & Troglodytes troglodytes & ökörszem & ökörszem & $5-40$ \\
\hline 32 & Phoenicurus ochruros & - & $\begin{array}{l}\text { rozsdafarkú, vörösbögy } \\
\text { (sic!), mákhasogató } \\
\text { pintyőke (sic!) }\end{array}$ & $40-60$ \\
\hline 33 & Turdus merula & feketerigó, rigó & feketerigó, rigó, szarmadár & $60-95$ \\
\hline 34 & Motacilla alba & $\begin{array}{l}\text { barázdabillegető, } \\
\text { brázdabillegető, } \\
\text { bráznobillegető }\end{array}$ & barázdabillegető & $5-40$ \\
\hline 35 & Motacilla flava & $*$ & - & $\leq 5$ \\
\hline 36 & Erithacus rubecula & vörösbögy & vörösbögy & $5-40$ \\
\hline 37 & Parus major, P. caeruleus & cinege, cinke, kékcinke & cinke & $60-95$ \\
\hline 38 & Parus caeruleus & - & cinke & $\leq 5$ \\
\hline 39 & Delichon urbicum & föcske, fecske & fecske, föcske & $5-40$ \\
\hline 40 & Hirundo rustica & föcske, fecske & fecske, föcske, füsti fecske & $5-40$ \\
\hline 41 & Riparia riparia & - & partifecske & $\leq 5$ \\
\hline 42 & Oriolus oriolus & sárgarigó & sárgarigó & $5-40$ \\
\hline 43 & Lanius collurio & szarkagáborján, gáborgyán & - & $\leq 5$ \\
\hline 44 & Sturnus vulgaris & seregély & seregély & $60-95$ \\
\hline 45 & Pica pica & szarka & szarka & $\geq 95$ \\
\hline 46 & Garrulus glandarius & szajkó & szajkó & $5-40$ \\
\hline
\end{tabular}




\begin{tabular}{|l|l|l|l|l|}
\hline & scientific name & local name in Slovenia & local name in Hungary & $\%$ \\
\hline 47 & Corvus frugilegus & csóka, feketecsóka & $\begin{array}{l}\text { csóka, fekete csóka, vetési } \\
\text { varjú }\end{array}$ & $60-95$ \\
\hline 48 & Corvus cornix & varjú, kánya & $\begin{array}{l}\text { varjú, kánya, vari, } \\
\text { dolmányos varjú }\end{array}$ & $\geq 95$ \\
\hline 49 & Corvus monedula & vari, varjú, káska, kavka & - & $5-40$ \\
\hline 50 & Corvus corax & holló & holló & $5-40$ \\
\hline 51 & $\begin{array}{l}\text { Passer domesticus, } \\
\text { P. montanus }\end{array}$ & veréb & veréb, csuri & $\geq 95$ \\
\hline 52 & Fringilla coelebs & pintyöke & - & $\leq 5$ \\
\hline 53 & Serinus serinus & - & sármány & $\leq 5$ \\
\hline 54 & Carduelis chloris & - & zöldike & $\leq 5$ \\
\hline 55 & Carduelis carduelis & - & tiglice & $\leq 5$ \\
\hline 56 & $\begin{array}{l}\text { Coccothraustes } \\
\text { coccothraustes }\end{array}$ & mákhasogató pintyöke & meggyvágó & $\leq 5$ \\
\hline 57 & Emberiza calandra & sördin, sordiny & - & $\leq 5$ \\
\hline
\end{tabular}

\section{DISCUSSION}

Compared with other regions (Kovács 1987; Gub 1996; BABAI 2011; UlicsNi ET AL. 2016), the number of folk taxa recorded in the examined regions demonstrates the existence of significant, detailed knowledge. The proportion of invertebrate to vertebrate taxa (55\% vs. $45 \%)$ is also similar to that found in the 1996 study by Gub (48\% vs. $52 \%$ ). Knowledge of some taxa was surprising and difficult to predict, since they had no particularly significant salient features (e.g. the spotted sulfur moth Emmelia trabealis; or sailor butterflies [Neptis spp.]). In the case of these species, we did not consider cultural salience to be likely either, although historical existence cannot be excluded. There were also similar proportions of species where it was difficult to explain the reason for their being known (KovÁcs 1987; GuB 1996) (e.g. the harvest mite: Microtrombidium spp.; and the two-spotted ladybug: Adalia bipunctata etc.). Knowledge of the vast majority of taxa, however, was easy to predict: based on their higher salience values and in keeping with our preliminary expectations, the majority of these taxa were well known in both regions. This is also confirmed by the $100 \%$ overlap between the two regions with respect to the list of known mammal taxa.

Due to the greater diversity of the butterfly fauna (Lepidoptera) in the Örség region compared to the Carpathian Basin, along with the existence of several rare and highly protected species, they are particularly significant from the point of view of conservation, thus they are also of importance in connection with nature conservation management measures, which represent a major source of conflict between farmers and conservationists. Similarly serious conflicts have emerged between local inhabitants and the authorities in relation to mammal species, arising from the opposition between the game husbandry and nature conservation values of these species, and the restrictions on activities that can be carried out by the local population and the losses these entail 
(Kozorog 2019). In relation to butterflies, the main sources of conflict are regulations concerning mowing times, which the national park has imposed taking into account the life cycle of the butterflies rather than the quality of the hay, which is the primary consideration among local farmers. In the case of big game animals, the most significant source of conflict - the direct damage caused by the trampling and eating of crops - is not primarily between nature conservationists and the local population, but rather between the local people and the game breeders.

The more ambitious nature conservation activities and the more active involvement of the local population in Hungarian culture clearly explain why, among the Hungarian folk names, there were a far bigger number (although, even so, amounting to a tiny minority) of official names originating from the media and from nature conservation professionals, rather than old folk names. In our experience, based on the origin of the name there is a good chance of being able to deduce the origin of the knowledge connected to it (in the case of taxa referred to by their professional names, knowledge originating from the media or from professional nature conservationists was generally significant). This phenomenon is also common in Göcsej (BAZSIKA 2010), for example in the case of the European stag beetle (Lucanus cervus) and dragonflies (Odonata). Knowledge of certain species that are especially important from a nature conservation point of view (e.g. the Corncrake [Crex crex] ) is undoubtedly owing to nature conservation communications. The agricultural restrictions imposed due to these species, which were previously unknown to the local population, are responsible for the majority of the conflicts.

Some genuine surprises are occasionally to be found in terms of taxon names. Some of the interviewees consistently referred to the Eurasian beaver (Castor fiber) as an otter, due to an old name being passed over to a new species (generally these interviewees were not aware of the Eurasian otter). Another striking example was the Barn Owl (Tyto alba) being referred to as a "snowy owl" which was an example of a new name, originating from the media, being passed over to an earlier known species.

The naming of many species clearly also refers to the species' most salient feature. In the case of earwigs (Dermaptera), for example, the reference is to the associated folklore - that is, that the insect makes its home in the human ear. Likewise, in the case of nocturnal moths (vacsoravesztö, or "dinner spoilers"), the reference is to their typical behavior - namely, that they are drawn inside by the light in the evening and occasionally fall into the food (cf. Kovícs 1987).

In other cases, it is far harder to draw inferences from the name, and certain welldifferentiated folk taxa are not even differentiated based on their names. Three folk taxa are simply referred to as "moths" even though they are distinctly separate in terms of their features. This phenomenon is found throughout the world, and occasionally even appears in quite extreme forms: in one Matsés tribe in Peru, only a single local name was used by interviewees for more than 50 species of bat, despite the local people's clear and detailed knowledge of their differences (FLECK et al. 2002).

In addition to the bigger number of potentially knowable species, the list of known invertebrates indicating the most significant difference between the two regions can also be dramatically influenced by a few particularly observant interviewees. On such occasions, knowledge emerges of taxa that are not called by a precise name, but that are precisely observed (e.g. the poppy weevil Ceutorhynchus macula-alba; and "snout beetles" - Curculionidae etc.). 


\section{CONCLUSION}

Despite the typical decline in traditional ecological knowledge in Europe, these recently gathered data indicate that a rich, vibrant knowledge is still to be found among the Hungarian inhabitants who supplied information in the Örség region and neighboring Slovenian villages. The especially large number of recorded folk taxa, and the accurate knowledge required to differentiate between them, confirm that even today it is worth conducting investigations on this topic in East Central Europe in the interests of gaining knowledge of, and conserving, cultural and natural values. Such knowledge can form a bridge, helping to establish a common language in the context of conflicts between local communities and nature conservationists. Knowledge reveals the interests and preferences of the local community and makes them easier to understand; it explains why communities behave as they do, and the reasons behind the cultural or agricultural prejudices that happen to be linked with certain animal species. Such information can be of enormous help to the nature conservation bodies operating in the area by contributing to the establishment of closer cooperation in a cultural landscape, shaped and maintained through extensive land use, that represents nature conservation and cultural and esthetic values in equal measure. It is for precisely this reason that such knowledge, and the extensive land use that is built on it, can also contribute to the sustainable management of natural values, while the more efficient integration of such knowledge in decision making is an indispensable task for the future.

\section{ACKNOWLEDGEMENTS}

We would like to express our gratitude to all the people of Goričko and Örség who patiently and generously shared their knowledge with us. Thanks go to Antónia Tóth, István Szentirmay, Anasztázia Gál, and Tamás Bódis for providing the contacts. We would also like to thank Tiborné Ulicsni for transcribing our recordings. Among those who participated in the research, the work of Dániel Babai was supported by a Premium Postdoctoral Fellowship (PPD 008/2017) from the Hungarian Academy of Sciences, and the work of Viktor Ulicsni was supported by the National Research, Development, and Innovation Office project: Protected Areas along the Slovenian-Hungarian Border. Challenges of Cooperation and Sustainable Development" (SNN 126230).

\section{REFERENCES CITED}

Alves, Rômulo Romeu da Nóbrega

2012 Relationships between fauna and people and the role of ethnozoology in animal conservation. Ethnobiology and Conservation 1:2. doi: 10.15451/ ec2012-8-1.2-1-69

Ambrus, András - BÁNKUTI, Károly - Kovács, Tibor

1995 Az Örség szitakötő faunája (Odonata) [The Dragonfly (Odonata) Fauna of the Örség]. Savaria - A Vas Megyei Múzeumok értesitóje 22(2):49-62. 
BABAI, Dániel

2011 Hagyományos ökológiai tudás az etnozoológia tükrében - A gerinces állatok népi ismerete Hidegségben [Traditional ecological knowledge in the light of ethnozoology: Folk knowledge of vertebrates in Hidegség]. MSc dissertation, Pécsi Tudományegyetem Bölcsészettudományi Kar Néprajz - Kulturális Antropológia Tanszék, Pécs.

BABAI, Dániel - Ulicsni, Viktor - BIRó, Marianna - JuHÁsz, Erika - MolnáR, Zsolt 2019 „Az emberek nem tudják miféle. Még emlékezet se volt róluk!” Természetismeret-szerzési mechanizmusok egy visszatelepített ökoszisztémamérnök faj (eurázsiai hód - Castor fiber) kapcsán [“People Don’t Know what Kind It Is. There Was Not Even a Memory of Them!" Mechanisms of Knowledge Acquisition in Relation to a Reintroduced Ecosystem Engineer Species (Eurasian Beaver - Castor Fiber)]. Ethno-Lore: A Magyar Tudományos Akadémia Néprajzi Kutatóintézetének Évkönyve 36:227-244.

BÁLINT, Zsolt

1994 Magyarország lepkéi a természetvédelem tükrében [The Butterflies of Hungary in the Scope of Nature Conservation]. Somogyi Múzeumok Közleményei 10:182-206.

BARTHA, Dénes

2016 Az Örségi Nemzeti Park élőhelytípusai [Habitat Types in the Örség National Park]. In BarthA, Dénes (ed.) Az Örségi Nemzeti Park I, 291-339. Öriszentpéter: Örségi Nemzeti Park.

BAZsIKA, Enikő

2010 Népi növény- és állatnevek Göcsejben [Folk Plant and Animal Names in Göcsej]. Muratáj Lendva - Lendava 2009(1-2):77-96.

BENíTEZ, Guillermo

2011 Animals used for medicinal and magico-religious purposes in western Granada Province, Andalusia (Spain). Journal of Ethnopharmacology 137(3):11131123.

Bentley, Jeffrey W - Rodríguez, Gonzalo

2001 Honduran Folk Entomology. Current Anthropology 42(2):285-301.

BERKES, Fikret

2012 Sacred ecology. 3rd ed. New York: Routledge.

BERLIN, Brent

2014 Ethnobiological classification: Principles of categorization of plants and animals in traditional societies. Princeton: Princeton University Press.

Brown, Cecil H.

1979 Folk Zoological Life-Forms: Their Universality and Growth. American Anthropologist, New Series 81(4):719-781.

Ceríaco, Luis M. P.

2012 Human attitudes towards herpetofauna: The influence of folklore and negative values on the conservation of amphibians and reptiles in Portugal. Journal of Ethnobiology and Ethnomedicine 8:8. https://doi.org/10.1186/1746-4269-8-8

Costa-Neto, Eraldo Medeiros

1998 Healing with animals in Feirade Santana City, Bahia, Brazil. Journal of Ethnopharmacology 65:225-230. 
2006 "Cricket singing means rain": semiotic meaning of insects in the district of Pedra Branca, Bahia State, Northeastern Brasil. Anais da Academia Brasileria de Cienicas 78(1): 59-68.

DiAmOND, Jared M. - Bishop, Kathryn D.

1999 Ethno-ornitology of Ketengban people Indonesian New Guinea. In Medin, Douglas L. - Atran, Scott (eds.) Folkbiology, 17-45. Cambridge: The MIT Press.

DövÉNYI, Zoltán

2010 Magyarország Kistájainak Katasztere [Cadastre of Microregions in Hungary]. Budapest: MTA Földrajztudományi Kutatóintézet.

ELLEN, Roy

2006 The cultural relations of classification: an analysis of Nuaulu animal categories from central Seram (Cambridge Studies in Social and Cultural Anthropology, Series Number 91). Cambridge: Cambridge University Press.

ESTABROOK, George F

2008 The significance of sheep in the traditional agriculture of Beira Alta, Portugal. Journal of Ethnobiology 4:55-68.

Fleck, David W - Voss, Robert S - Simmons, Nancy B

2002 Underdifferentiated taxa and sublexical categorization: an example from Matses classification of bats. Journal of Ethnobiology 22(1):61-102.

Fridell, Staffan - SvanBerg, Ingvar

2007 Däggdjur i svensk folklig tradition. Dialogos Förlag.

GuB, Jenő

1996 Erdö-mezö állatai a Sóvidéken [Woodland and Meadow Animals in Sóvidék]. Korond: Firtos Mủvelödési Egyesület.

Hahn, Anke - Konkoly-Gyuró, Éva - Völler, Sonja - Balázs, Pál - Torkar, Gregor - BURNET, Ellis Burnet

2012 Perception of Landscape Changes in Three Trans-boundary Focus Areas: Based on Oral History Surveys with Local Inhabitants, Stakeholders and Experts. Győr: University of West Hungary.

Hemp, Claudia

2001 Ethnozoological research on invertebrates on Mt. Kilimanjaro, Tanzania. Ecotropica 7(1-2):139-149.

Hewlett, Barry S. - CAVAlLi-Sforza, Luigi Luca

1986 Cultural Transmission among Aka Pygmies. American Anthropologist 88:922934.

HoppáL, Mihály

1982 Népi természetismeret [Folk knowledge of nature]. In OrTUTAY, Gyula (ed.)

HunN, Eugene S. Néprajzi Lexikon V., 271. Budapest: Akadémiai.

1982 The Utilitarian Factor in Folkbiological Classification. American Anthropologist, New Series 84(4):830-847.

1999 Size as Limiting the Recognition of Biodiversity in Folkbiological Classifications: One of Four Factors Governing the Cultural Recognition of Biological Taxa. In Medin, Douglas L. - Atran, Scott (eds.) Folkbiology, 47-70. Cambridge: The MIT Press. 
Ispén, Ágota Lídia - BABAI, Dániel - MóD, László - Ulicsni, Viktor - MÉsZÁros, Csaba 2018 Complex Ethnographic Research Methods for the Study of Protected Areas and Border Communities at the Slovenian-Hungarian Border. Acta Ethnographica Hungarica 63(2):471-500.

Kaligarič, Mitja - SedonJa, Jožef - Šajna, Nina

2008 Traditional Agricultural Landscape in Goričko Landscape Park (Slovenia): Distribution and Variety of Riparian Stream Corridors and Patches. Landscape and Urban Planning 85(1):71-78.

Kovács, Antal

1987 “Járok-kelek gyöngyharmaton...” Növény- és állatnevek a Felső-Szigetköz tájnyelvében ["Walking on Pearls of Dew". Plant and Animal Names in the Dialect of Felsö-Szigetköz]. Mosonmagyaróvári Helytörténeti Füzetek 6.

Kozorog, Miha

2019 "They feed here and live there": Borderwork with wildlife in Slovenia's north-east corner "Tu se hranijo, tam pa živijo": Izdelovanje meje z divjadjo v severovzhodnem kotu Slovenije. Traditiones 48(1):191-211.

KUTALEK, Ruth - Kassa, Afework

2005 The use of Gyrinids and Dytiscids for stimulating breast growth in East Africa. Journal of Ethnobiology 25(1):115-128.

LescureuX, Nicolas - LinNeLl, John D. C.

2010 Species specific ecology influences the perceptions and local knowledge of Macedonian hunters and livestock breeders towards bears, wolves and lynx. Human Ecology 38(3):289-299.

REA, Amadeo M.

1998 Folk Mammalogy of the Northern Pimans. Tucson: University of Arizona Press.

2007 Wings in the desert. A Folk Ornithology of the Northern Pimans. Tucson: University of Arizona Press.

SVANBERG, Ingvar

2006 Black slugs (Arion ater) as grease: a case study of technical use of Gastropods in pre-induustrial Sweden. Journal of Ethnobiology 26(2):299-309.

UliCSNI, Viktor - BABAI, Dániel - JuHÁsz, Erika - MolnáR, Zsolt - BIRó, Marianna

2020 A loveable nuisance: local knowledge of the Eurasian beaver (Castor fiber) and perception of its impact on ecosystem services. PLOS ONE 15(5):e0233506. https://doi.org/10.1371/journal.pone.0233506

Ulicsni, Viktor - BABAI, Dániel - VADÁsZ, Csaba - VADÁsZ-BeSNYöI, Vera - BÁLDI, András - MoLNÁR, Zsolt

2019 Bridging conservation science and traditional knowledge of wild animals: The need for expert guidance and inclusion of local knowledge holders. Ambio 48(7):769-778.

UlicSNI, Viktor - SvANBERG, Ingvar - MolnÁR, Zsolt

2016 Folk knowledge of invertebrates in Central Europe-folk taxonomy, nomenclature, medicinal and other uses, folklore, and nature conservation. Journal of Ethnobiology and Ethnomedicine 12(1):47. https://doi.org/10.1186/ s13002-016-0118-7 
VíG, Károly

1999 Vas megye élővilágának megismerése, jelenkori természeti értékei [Wildlife and Its Current Natural Values in Vas County]. Savaria - A Vas Megyei Múzeumok Értesitöje 25(2):7-82.

2003 Zoological Research in Western Hungary. Szombathely: Vas County Body of the Hungarian Academy of Sciences.

WALKER, Danny N.

2000 Preliminary bibliography on dogs and wolves stressing their prehistoric and historic occurence, hybridisation and domestication. British Archaeological Reports (BAR) International Series 23:313-343.

WePUKHUlu, David Masika

1992 Bukusu Ethnozoology (Invertebrates) - II. Mombasa, Kenya: University of Kenya. https://www.scribd.com/collections/2316838/Bukusu. Accessed October 142020.

ZENT, Stanford

2001 Acculturation and ethnobotanical knowledge loss among the Piaroa of Venezuela: Demonstration of a quantitative method for the empirical study of TEK change. In MAFFI, Luisa (ed.) On biocultural diversity: Linking language, knowledge, and the environment, 190-211. Washington: Smithsonian Institution Press.

Viktor Ulicsni is a research fellow at the Centre for Ecological Research, Institute of Ecology and Botany. He is an ethnozoologist and ecologist working in the Carpathian Basin and Moldova. E-mail: ulicsni.viktor@ecolres.hu

Dániel Babai is a research fellow at the Institute of Ethnology, Research Centre for the Humanities (ELKH). As an ethnoecologist, trained in both biology and cultural anthropology, he is interested in human-nature interactions, especially in extensive land-use management, sustainable forms of natural resource management, and related traditional ecological knowledge in the Carpathian Basin. E-mail: babai.daniel@btk. mta.hu

Open Access. This is an open-access article distributed under the terms of the Creative Commons Attribution 4.0 International License (https://creativecommons.org/licenses/by/4.0), which permits unrestricted use, distribution, and reproduction in any medium, provided the original author and source are credited, a link to the CC License is provided, and changes - if any - are indicated. (SID_1) 